\title{
Sodium nitroprusside infusion after bidirectional superior cavopulmonary connection: Preserved cerebral blood flow velocity and systemic oxygenation
}

Janet M. Simsic, MD

Scott M. Bradley, MD ${ }^{\mathrm{b}}$

Denise M. Mulvihill, MD ${ }^{\mathrm{c}}$

From the Divisions of Pediatrics, ${ }^{\text {a }}$ Cardiology, ${ }^{\mathrm{b}}$ Cardiothoracic Surgery, and Radiology, ${ }^{\mathrm{c}}$ Medical University of South Carolina, Charleston, SC.

Received for publication Sept 18, 2002; revisions requested Nov 4, 2002; revisions received Jan 2, 2003; accepted for publication March 14, 2003.

Address for reprints: Janet M. Simsic, MD, Sibley Heart Center Cardiology, 52 Executive Park South, Suite 5200, Atlanta, GA 30329 (E-mail: simsicj@kidsheart.com).

J Thorac Cardiovasc Surg 2003;126:186-90

Copyright $(\odot) 2003$ by The American Association for Thoracic Surgery

$0022-5223 / 2003 \$ 30.00+0$

doi:10.1016/S0022-5223(03)00582-8
Objective: Systemic hypertension is common in patients after bidirectional superior cavopulmonary connection. It can be treated with a vasodilator, such as sodium nitroprusside. However, it is possible that systemic hypertension is necessary to maintain cerebral blood flow in the face of cerebral venous hypertension. Furthermore, bidirectional superior cavopulmonary connection places the cerebral and pulmonary vascular beds in series. Thus treatment of systemic hypertension by lowering blood pressure might decrease cerebral blood flow, pulmonary blood flow, and systemic oxygen levels. The aim of the current study was to determine the effects of sodium nitroprusside on cerebral blood flow velocity and systemic oxygenation in patients after bidirectional superior cavopulmonary connection.

Methods: This is a prospective patient-controlled study of 9 patients (median age, 7 months; age range, 4 to 12 months) undergoing bidirectional superior cavopulmonary connection. Patients were studied in the intensive care unit within 6 hours of surgical intervention while sedated, paralyzed, and mechanically ventilated. Sodium nitroprusside was infused to achieve a decrease in mean systemic blood pressure of approximately 20\%. Cerebral blood flow velocity was measured by means of transcranial Doppler ultrasonography of the middle cerebral artery.

Results: During sodium nitroprusside infusion, mean systemic blood pressure decreased (from $69 \pm 6 \mathrm{~mm} \mathrm{Hg}$ at baseline to $58 \pm 6 \mathrm{~mm} \mathrm{Hg}, P<.05$ ). However, there was no accompanying change in 2 indicators of cerebral blood flow: blood flow velocity in the middle cerebral artery and arteriovenous oxygen saturation difference across the upper body. Both arterial $\mathrm{Po}_{2}$ and systemic oxygen saturation were also preserved during sodium nitroprusside infusion.

Conclusions: Sodium nitroprusside decreases systemic blood pressure in patients after bidirectional superior cavopulmonary connection. This decrease occurs without accompanying changes in cerebral blood flow velocity or systemic oxygen levels. These findings suggest that cerebral and pulmonary blood flows are preserved during sodium nitroprusside infusion. Sodium nitroprusside appears to be an appropriate agent for the treatment of systemic hypertension after bidirectional superior cavopulmonary connection.

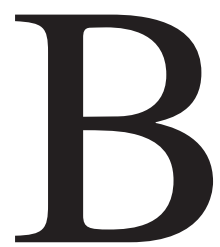

idirectional superior cavopulmonary connection (BSCC) is widely used in patients with functional single ventricles. The postoperative course after BSCC is frequently characterized by systemic hypertension, which can be treated with a vasodilator, such as sodium nitroprusside. ${ }^{1}$ The cause of systemic hypertension after BSCC is unknown. BSCC increases superior vena caval pressure and therefore cerebral venous pressure. It is possible that systemic hypertension is necessary to maintain the transcerebral pressure gradient and therefore cerebral blood flow in 
the face of cerebral venous hypertension. Treatment of systemic hypertension by lowering blood pressure might then decrease cerebral blood flow. The effect of lowering blood pressure after BSCC on cerebral blood flow has not been previously examined.

Furthermore, BSCC places the cerebral and pulmonary vascular beds in series. Previous studies have shown that after BSCC, interventions that alter cerebral blood flow, such as hyperventilation and hypoventilation, also alter pulmonary blood flow and systemic oxygenation. ${ }^{2,3}$ Thus treatment of systemic hypertension might decrease not just cerebral blood flow but also pulmonary flow and systemic oxygen levels. The aim of the current study was to determine the effects of sodium nitroprusside on cerebral blood flow velocity and systemic oxygenation in patients after BSCC.

\section{Methods \\ Patients}

This study was approved by the Institutional Review Board of the Medical University of South Carolina; informed consent was obtained for all patients. Nine patients undergoing BSCC were prospectively studied. The median patient age was 7 months (range, 4-12 months), and the median weight was $7.0 \mathrm{~kg}$ (range, 4.9-10.0 $\mathrm{kg}$ ). There were 4 male and 5 female patients. Diagnoses were hypoplastic left heart syndrome (3), double-inlet left ventricle (2), tricuspid atresia (1), double-outlet right ventricle with mitral atresia (1), pulmonary atresia with intact ventricular septum (1), and crisscross heart with straddling atrioventricular valves (1). Previous operations were a Norwood procedure in 5 patients, modified Blalock-Taussig shunt placement in 3 patients, and none in 1 patient. At preoperative cardiac catheterization in 8 patients, pulmonary venous wedge pressure was $16 \pm 4 \mathrm{~mm} \mathrm{Hg}$, transpulmonary gradient was $7 \pm 1 \mathrm{~mm} \mathrm{Hg}$, and pulmonary vascular resistance was $2.0 \pm 0.4$ units $/ \mathrm{m}^{2}$. No patient had significant aortopulmonary collaterals identified at preoperative angiography.

\section{Surgical Techniques}

BSCC consisted of a bidirectional Glenn shunt in 8 patients and a hemi-Fontan procedure in 1 patient. ${ }^{1,4-6}$ All systemic to pulmonary shunts were divided at the time of BSCC; therefore the BSCC provided the only source of pulmonary blood flow in all patients. Concomitant procedures included pulmonary arterioplasty in 5 patients, atrial septectomy in 4 patients, aortic valve repair in 1 patient, and innominate artery reimplantation in 1 patient. All operations were performed through a median sternotomy and used cardiopulmonary bypass (144 \pm 49 minutes). Myocardial cardioplegic arrest was used in 6 patients $(28 \pm 15$ minutes $)$, and circulatory arrest was used in 4 patients (16 \pm 6 minutes).

\section{Study Protocol}

All patients were studied in the pediatric cardiac intensive care unit within 6 hours of surgical intervention while sedated (fentanyl, 20 $\mu \mathrm{g} \cdot \mathrm{kg}^{-1} \cdot \mathrm{h}^{-1}$ ), paralyzed (vecuronium, $0.1 \mathrm{mg} \cdot \mathrm{kg}^{-1} \cdot \mathrm{h}^{-1}$ ), and mechanically ventilated. At the time of the study, all patients were receiving milrinone infusions $\left(0.5-0.75 \mu \mathrm{g} \cdot \mathrm{kg}^{-1} \cdot \mathrm{min}^{-1}\right) ; 5$ were also receiving dopamine infusions $\left(3-10 \mu \mathrm{g} \cdot \mathrm{kg}^{-1} \cdot \mathrm{min}^{-1}\right)$. All infusions were maintained at constant rates, and no blood transfusions were given during the study. Three of the 9 patients received a single bolus of $5 \%$ albumin $(5 \mathrm{~mL} / \mathrm{kg})$ during the protocol. All patients were in normal sinus rhythm throughout the study. Patients were mechanically ventilated with a Servo 300 ventilator (Siemens-Elema AB, Solna, Sweden) in pressure-regulated volume control mode. The fraction of inspired oxygen was set at 1.0, peak end-expiratory pressure at $0 \mathrm{~cm} \mathrm{H}_{2} \mathrm{O}$, and tidal volume at 14 to $18 \mathrm{~mL} / \mathrm{kg}$; the ventilator rate was adjusted to achieve a normal $\mathrm{pH}$ and $\mathrm{PCO}_{2}$.

Patients were studied at baseline (time 1), during sodium nitroprusside infusion (time 2), and after return to baseline (time 3). Sodium nitroprusside was titrated to achieve a decrease in the mean arterial blood pressure of approximately $20 \%$ (median dose, $3 \mu \mathrm{g} \cdot \mathrm{kg}^{-1} \cdot \mathrm{min}^{-1}$; range, $1-4 \mu \mathrm{g} \cdot \mathrm{kg}^{-1} \cdot \mathrm{min}^{-1}$ ). Once the target blood pressure was obtained, sodium nitroprusside was continued for a 15-minute stabilization period before determination of arterial blood gas, hemodynamic, and transcranial cerebral Doppler values. Arterial blood gases were obtained from samples drawn from radial or femoral arterial catheters. Systemic oxygen saturation was measured by means of extremity oximetry (HewlettPackard Co, Palo Alto, Calif). Common atrial and pulmonary artery pressures were measured through transthoracic lines placed in the operating room. The transpulmonary gradient was derived as pulmonary artery pressure minus common atrial pressure. Pulmonary artery oxygen saturation was determined from samples from the pulmonary artery line. The upper body arteriovenous oxygen saturation difference was derived as the systemic minus the superior vena caval (pulmonary artery) oxygen saturation.

Cerebral blood flow velocity was determined by means of transcranial Doppler ultrasonography of the middle cerebral artery by using a temporal window. The middle cerebral artery was identified by means of color flow Doppler ultrasonography and a maximum signal recorded from the M1 segment by using a 4-MHz transducer. The time-averaged maximum velocity acquired over a 1-minute period was used for analysis. Studies were performed with either an Acuson 128 or Acuson Sequoia machine (Siemens).

\section{Statistics}

Data are shown as means \pm SD. Comparison of the 3 time points in the study protocol was by means of repeated-measures analysis of variance. Multiple comparison testing was done with paired $t$ tests with the Bonferroni correction. In our 2 previous studies of infants after BSCC, changes in ventilation (hyperventilation or hypoventilation) produced changes of $27 \%$ to $49 \%$ from baseline in cerebral flow velocity and of 20 to $35 \%$ in systemic $\mathrm{Po}_{2} .{ }^{2,3} \mathrm{On}$ the basis of those studies, we considered a change of at least $30 \%$ from baseline cerebral flow velocity and at least $20 \%$ from baseline systemic $\mathrm{PO}_{2}$ to be important. Sample size calculations indicated that a sample size of 8 patients would be required to show such differences from the baseline values in this study, with an $\alpha$ value of .05 and a $\beta$ value of .2 (power of $80 \%$ ).

\section{Results}

Sodium nitroprusside lowered systemic blood pressure but had no significant effect on other hemodynamic parameters (Table 1). During infusion of sodium nitroprusside, mean 
TABLE 1. Hemodynamic parameters

\begin{tabular}{lccc}
\hline & $\begin{array}{c}\text { Time 1: } \\
\text { Baseline }\end{array}$ & $\begin{array}{c}\text { Time 2: Sodium } \\
\text { nitroprusside }\end{array}$ & $\begin{array}{c}\text { Time 3: } \\
\text { Baseline }\end{array}$ \\
\hline $\begin{array}{c}\text { Systemic blood } \\
\text { pressure (mm Hg) }\end{array}$ & & & \\
$\quad$ Systolic & $98 \pm 9$ & $84 \pm 11^{*}$ & $98 \pm 7$ \\
$\quad$ Diastolic & $53 \pm 5$ & $46 \pm 7^{*}$ & $53 \pm 6$ \\
$\quad$ Mean & $69 \pm 6$ & $58 \pm 6^{*}$ & $68 \pm 6$ \\
$\begin{array}{c}\text { Pulmonary artery } \\
\text { pressure (mm Hg) }\end{array}$ & $13 \pm 2$ & $12 \pm 2$ & $13 \pm 2$ \\
$\begin{array}{c}\text { Common atrial } \\
\text { pressure (mm Hg) }\end{array}$ & $7 \pm 1$ & $6 \pm 1$ & $7 \pm 1$ \\
$\begin{array}{c}\text { Transpulmonary } \\
\text { gradient (mm Hg) }\end{array}$ & $6 \pm 2$ & $6 \pm 2$ & $6 \pm 2$ \\
$\begin{array}{c}\text { Heart rate } \\
\text { (beats/min) }\end{array}$ & $142 \pm 14$ & $143 \pm 13$ & $141 \pm 12$ \\
\hline
\end{tabular}

${ }^{*} P<.05$ vs. baseline (analysis of variance).

systemic blood pressure decreased from $69 \pm 6$ to $58 \pm 6$ $\mathrm{mm} \mathrm{Hg}(P<.05)$. This decrease was not accompanied by changes in pulmonary artery pressure, common atrial pressure, transpulmonary gradient, or heart rate.

At baseline, the arterial $\mathrm{Po}_{2}$ value was $49 \pm 12 \mathrm{~mm} \mathrm{Hg}$, and systemic oxygen saturation was $84 \% \pm 7 \%$ (Table 2). Sodium nitroprusside infusion had no significant effect on either measure of systemic oxygenation (Table 2).

Two indicators of cerebral blood flow were examined: blood flow velocity in the middle cerebral artery and arteriovenous oxygen saturation difference across the upper body. At baseline, flow velocity in the middle cerebral artery was $54 \pm 16 \mathrm{~cm} / \mathrm{s}$, and the upper body arteriovenous oxygen saturation difference was $32 \% \pm 9 \%$. Neither parameter changed during infusion of sodium nitroprusside (Table 3).

\section{Discussion}

Systemic hypertension is frequently observed after BSCC. Chang and colleagues ${ }^{1}$ reviewed the postoperative course and early results of BSCC in young infants at Children's Hospital, Boston. They found systolic hypertension in $88 \%$ of their patients. One half of those were treated with a vasodilator (either sodium nitroprusside or amrinone). The severity of systolic hypertension decreased in all patients after the first 24 postoperative hours. However, almost 50\% were discharged from the hospital on a regimen of antihypertensive medication.

The cause of systemic hypertension after BSCC is unknown. Potential contributing factors are postoperative pain and issues unique to the BSCC, such as hormone levels, altered ventricular geometry, and increased cerebral venous pressure. Increased levels of antidiuretic hormone, cortisol, and aldosterone have been documented in the first day after BSCC. ${ }^{7}$ Increases in antidiuretic hormone, angiotensin II,
TABLE 2. Arterial blood gas values

\begin{tabular}{lccc}
\hline & $\begin{array}{c}\text { Time 1: } \\
\text { Baseline }\end{array}$ & $\begin{array}{c}\text { Time 2: Sodium } \\
\text { nitroprusside }\end{array}$ & $\begin{array}{c}\text { Time 3: } \\
\text { Baseline }\end{array}$ \\
\hline $\mathrm{PO}_{2}(\mathrm{~mm} \mathrm{Hg})$ & $49 \pm 12$ & $47 \pm 9$ & $45 \pm 10$ \\
$\mathrm{PCO}_{2}(\mathrm{~mm} \mathrm{Hg})$ & $41 \pm 4$ & $40 \pm 5$ & $41 \pm 6$ \\
$\mathrm{pH}$ & $7.40 \pm 0.03$ & $7.40 \pm 0.05$ & $7.40 \pm 0.1$ \\
$\mathrm{Bicarbonate}$ & $25 \pm 2$ & $24 \pm 2$ & $25 \pm 2$ \\
$\quad$ (mmol/L) & & $81 \pm 6$ & $81 \pm 8$ \\
$\begin{array}{c}\text { Systemic oxygen } \\
\text { saturation (\%) }\end{array}$ & $84 \pm 7$ & & \\
\hline
\end{tabular}

TABLE 3. Cerebral blood flow indicators

\begin{tabular}{lccc}
\hline & $\begin{array}{c}\text { Time 1: } \\
\text { Baseline }\end{array}$ & $\begin{array}{c}\text { Time 2: Sodium } \\
\text { nitroprusside }\end{array}$ & $\begin{array}{c}\text { Time 3: } \\
\text { Baseline }\end{array}$ \\
\hline $\begin{array}{l}\text { Middle cerebral artery } \\
\text { flow velocity (cm/s) }\end{array}$ & $54 \pm 16$ & $54 \pm 11$ & $58 \pm 18$ \\
$\begin{array}{l}\text { Upper body } \\
\text { arteriovenous } \\
\text { oxygen saturation } \\
\text { difference (\%) }\end{array}$ & $32 \pm 9$ & $33 \pm 9$ & $33 \pm 8$ \\
\hline
\end{tabular}

renin, and atrial natriuretic factor have also been observed late $\left(2\right.$ years) after BSCC. ${ }^{8}$ Increases in these or other hormones could contribute to systemic hypertension. After BSCC, reduction in ventricular volume load results in altered ventricular geometry. This is characterized by an acute decrease in ventricular end-diastolic volume and an increase in wall thickness. ${ }^{9}$ It is also possible that altered ventricular geometry and ejection characteristics could contribute to systemic hypertension.

A third issue unique to the BSCC is cerebral venous hypertension. By connecting the superior vena cava to the pulmonary arteries, BSCC results in increased superior vena caval pressure and therefore cerebral venous pressure. It has been hypothesized that systemic hypertension is a compensatory reflex aimed at maintaining transcerebral pressure gradient and cerebral blood flow in the face of cerebral venous hypertension. ${ }^{1}$ If this hypothesis is correct, treating systemic hypertension after BSCC by lowering blood pressure could decrease cerebral blood flow. The effect of lowering blood pressure after BSCC on cerebral blood flow has not been previously examined.

In the current study sodium nitroprusside had no significant effect on 2 indicators of cerebral blood flow, cerebral blood flow velocity in the middle cerebral artery and upper body arteriovenous oxygen saturation difference. The validity of using middle cerebral artery flow velocity as an indicator of cerebral blood flow has been previously discussed. ${ }^{2,3,10}$ Because flow through a vessel is equal to mean flow velocity multiplied by cross-sectional area, changes in flow velocity reflect changes in flow as long as the vessel 
diameter does not change. In children during cardiopulmonary bypass, the diameter of the middle cerebral artery has been found to remain constant as blood pressure changes. ${ }^{11}$ Furthermore, changes in middle cerebral artery flow velocity correlate with changes in cerebral flow, as measured on the basis of internal carotid artery flow volume, ${ }^{12}$ the KetySchmidt method, ${ }^{13}$ and xenon washout techniques. ${ }^{10,14}$ Correlation with xenon washout has been found both during and after hypothermic cardiopulmonary bypass. ${ }^{14}$ Transcranial Doppler ultrasonography of the middle cerebral artery is also an extremely sensitive method of detecting cerebral blood flow. During low-flow cardiopulmonary bypass in children, transcranial Doppler ultrasonography has been shown to detect middle cerebral artery flow, with reduction of the bypass pump flow as low as $30 \mathrm{~mL} / \mathrm{kg} .{ }^{15,16}$ In the present study blood flow velocity in the middle cerebral artery was unchanged during sodium nitroprusside infusion. The absolute flow velocities measured were similar to those seen in previous studies of pediatric patients after cardiopulmonary bypass. ${ }^{2,3,16}$ Thus the current findings are in line with those of previous studies and suggest that cerebral blood flow did not change during sodium nitroprusside infusion.

The second indicator of cerebral blood flow used in the current study was arteriovenous oxygen saturation difference across the upper body. By using the Fick principle, a decrease in cerebral and upper body blood flow would be expected to result in an increase in the upper body arteriovenous oxygen saturation difference. This difference did not change in our patients during sodium nitroprusside infusion. This finding is further consistent with preservation of cerebral blood flow during sodium nitroprusside infusion. It is also in agreement with the results of other studies that found no change in cerebral blood flow during infusion of nitroprusside in human subjects during sedation or anesthesia. ${ }^{17,18}$ BSCC provides pulmonary blood flow by connecting the superior vena cava to the pulmonary arteries. This connection places the cerebral and pulmonary vascular beds in series. Alterations in cerebral blood flow might therefore be expected to affect pulmonary blood flow and systemic oxygen levels in patients with a BSCC. In previous studies we found that alterations in arterial $\mathrm{PCO}_{2}$, which are known to affect cerebral blood flow, ${ }^{19}$ do affect systemic oxygenation after BSCC. ${ }^{2,3}$ Hyperventilation, which produces hypocarbia, reduced both flow velocity in the middle cerebral artery and systemic $\mathrm{Po}_{2}$ in patients after BSCC. ${ }^{2}$ Conversely, hypoventilation increased both middle cerebral artery flow velocity and systemic oxygenation. ${ }^{3}$ This linkage of cerebral and pulmonary blood flow raises the possibility that lowering blood pressure after BSCC might impair not just cerebral but also pulmonary blood flow. In the presence of intracardiac mixing of systemic and pulmonary venous return, as occurs after BSCC, decreased pulmonary blood flow will result in decreased systemic oxygen levels.

In the current study sodium nitroprusside had no significant effect on systemic oxygenation. Neither systemic $\mathrm{Po}_{2}$ nor oxygen saturation changed when systemic blood pressure was decreased by nitroprusside. This suggests that pulmonary blood flow, along with cerebral blood flow, was preserved during sodium nitroprusside infusion. This information might be particularly useful in the management of patients who are hypoxemic after $\mathrm{BSCC}^{5,20,21}$ : a vasodilator can be used to treat systemic hypertension without further compromising systemic oxygenation.

In our study patients sodium nitroprusside effectively reduced systemic blood pressure without causing other hemodynamic alterations. Systemic hypertension after cardiac surgery is undesirable for several reasons. Increased blood pressure can contribute to postoperative bleeding. It also increases ventricular afterload, which has the potential to decrease cardiac output. Increased ventricular afterload might be particularly undesirable in patients with decreased ventricular function caused by myocardial stunning early after cardiac surgery and in patients with single-ventricle palliation rather than biventricular repair. Our results suggest that sodium nitroprusside is an effective and safe treatment for systemic hypertension early after BSCC.

The current study was prospective and patient-controlled. Respiratory and cardiac variables, including inotrope delivery, were controlled so as to isolate the effects of sodium nitroprusside infusion. A limitation of the study is the small number of patients. However, because of the study design, in which each patient served as his or her own control subject, this number of patients would have allowed us to detect changes of $30 \%$ from baseline cerebral flow velocity and of $20 \%$ from baseline systemic $\mathrm{PO}_{2}$ with a power of $80 \%$. These changes are equivalent to those observed in our 2 previous studies of infants after BSCC. ${ }^{2,3}$ It remains possible that changes of smaller magnitude could have occurred during sodium nitroprusside infusion but not been detected in this study. A further study limitation is that neither cerebral nor pulmonary blood flow was directly measured because such measurements are difficult in infants early after cardiac surgery. Conditions of sedation and paralysis were used to eliminate spontaneous respiration and minimize response to stimulation, and all patients were receiving milrinone infusions at the time of the study. Whether these results would also apply in patients who were spontaneously ventilating or not already receiving a vasodilator would require further study.

In summary, sodium nitroprusside decreases systemic blood pressure in patients after BSCC. This decrease occurs without accompanying changes in cerebral blood flow velocity or systemic oxygen levels. These findings suggest that both cerebral and pulmonary blood flow are preserved 
during sodium nitroprusside infusion. Thus sodium nitroprusside appears to be an appropriate agent for the treatment of systemic hypertension after BSCC.

\section{References}

1. Chang AC, Hanley FL, Wernovsky G, Rosenfeld HM, Wessel DL, Jonas RA, et al. Early bidirectional cavopulmonary shunt in young infants. Circulation. 1993;88(Suppl II):II149-58.

2. Bradley SM, Simsic JM, Mulvihill DM. Hyperventilation impairs oxygenation after bidirectional superior cavopulmonary connection. Circulation. 1998;98(Suppl II):II372-7.

3. Bradley SM, Simsic JM, Mulvihill DM. Hypoventilation improves oxygenation after bidirectional superior cavopulmonary connection. $J$ Thorac Cardiovasc Surg. In press.

4. Hopkins RA, Armstrong BE, Serwer GA, Peterson RJ, Oldham NH. Physiologic rationale for a bidirectional cavopulmonary shunt. $J$ Thorac Cardiovasc Surg. 1995;90:391-8.

5. Bradley SM, Mosca RS, Hennein HA, Crowley DC, Kulik TJ, Bove EL. Bidirectional superior cavopulmonary connection in young infants. Circulation. 1996;94(Suppl II):II5-11.

6. Norwood WI, Jacobs ML. Fontan's procedure in two stages. Am J Surg. 1993;166:548-51.

7. Mainwaring RD, Lamberti JJ, Moore JW, Billman GF, Nelson JC. Comparison of the hormonal response after bidirectional Glenn and Fontan procedures. Ann Thorac Surg. 1994;57:59-64.

8. Hjortdal VE, Stenbog EV, Raven HB, Emmertsen K, Jensen KT, Pederson EB, et al. Neurohormonal activation late after cavopulmonary connection. Heart. 2000;83:439-43.

9. Rychik J, Jacobs ML, Norwood WI. Acute changes in left ventricular geometry after volume reduction operation. Ann Thorac Surg. 1995; 60:1267-74.

10. Bishop CCR, Powell S, Rutt D, Browse NL. Transcranial Doppler measurement of middle cerebral artery blood flow velocity: a validation study. Stroke. 1986;17:913-5.

11. van der Linden J, Priddy R, Ekroth R, Lincoln C, Pugsley W, Scallan $\mathrm{M}$, et al. Cerebral perfusion and metabolism during profound hypo- thermia in children; a study of middle cerebral artery ultrasonic variables and cerebral extraction of oxygen. J Thorac Cardiovasc Surg. 1991;102:103-14.

12. Lindegaard K, Lundar T, Wiberg J, Sjoberg D, Aaslid R, Nornes H. Variations in middle cerebral artery blood flow investigated with noninvasive transcranial blood velocity measurements. Stroke. 1987; 18:1025-30.

13. Weyland A, Stephan H, Grune F, Weyland W, Sonntag H. Effect of ketanserin on global cerebral blood flow and middle cerebral artery flow velocity. Anesth Analg. 1995;80:64-70.

14. Trivedi UH, Patel RL, Turtle MRJ, Venn GE, Chanbers DJ. Relative changes in cerebral blood flow during cardiac operations using xenon133 clearance versus transcranial Doppler sonography. Ann Thorac Surg. 1997;63:167-74.

15. Zimmerman AA, Burrows FA, Jonas RA, Zhickey PR. The limits of detectable cerebral perfusion by transcranial Doppler sonography in neonates undergoing deep hypothermic low-flow cardiopulmonary bypass. J Thorac Cardiovasc Surg. 1997;114:594-600.

16. Jonassen AE, Quaegebeur JM, Young WL. Cerebral blood flow velocity in pediatric patients is reduced after cardiopulmonary bypass with profound hypothermia. J Thorac Cardiovasc Surg. 1995;110: 934-43.

17. Joshi S, Young WL, Duong H, Aagaard BA, Ostapkovich ND, Connolly ES, et al. Intracarotid nitroprusside does not augment cerebral blood flow in human subjects. Anesthesiology. 2002;96:60-6.

18. Larson R, Teichmann J, Hilfiker O, Busse C, Sonntag H. Nitroprusside-hypotension: cerebral blood flow and cerebral oxygen consumption in neurosurgical patients. Acta Anesthesiol Scand. 1982;26:32730 .

19. Madden JA. The effect of carbon dioxide on cerebral arteries. Pharmacol Ther. 1993;59:229-50.

20. Slavik Z, Lamb RK, Webber SA, Devlin AM, Keeton BR, Monro JL, et al. Bidirectional superior cavopulmonary anastomosis: how young is too young? Heart. 1996;75:78-82.

21. Reddy VM, Liddicoat JR, Hanley FL. Primary bidirectional superior cavopulmonary shunt in infants between 1 and 4 months of age. Ann Thorac Surg. 1995;59:1120-6.

\section{Availability of Journal back issues}

As a service to our subscribers, copies of back issues of The Journal of Thoracic and Cardiovascular Surgery for the preceding 5 years are maintained and are available for purchase from Mosby until inventory is depleted. Please write to Mosby, Subscription Customer Service, 6277 Sea Harbor Dr, Orlando, FL 32877, or call $800-654-2452$ or $407-345-4000$ for information on availability of particular issues and prices. 